\title{
Albuminuria in the Elderly More than a Marker of Renal or Cardiovascular Disease
}

\author{
Joshua I Barzilay ${ }^{1 *}$ and Kenneth J Mukamal ${ }^{2}$ \\ ${ }^{1}$ Kaiser Permanente of Georgia, Atlanta, Georgia \\ ${ }^{2}$ Department of Medicine, Beth Israel Deaconess Medical Center, Harvard University, Boston, MA
}

*Corresponding author: Joshua I Barzilay, Kaiser Permanente of Georgia, 3650

Steve Reynolds Blvd, Duluth, GA 30096.

Received Date: June 25, 2020

Published Date: July 17, 2020

\section{Background}

Albuminuria - the presence of more than $30 \mathrm{mg}$ of alumin per one gram of creatinine in the urine - is highly prevalent in older adults. Based on data from the NHANES III study, more than $20 \%$ of adults over the age of 70 years have albuminuria, while among similarly aged adults with diabetes the prevalence reaches $40 \%$ $[1,2]$. To date, most studies have examined albuminuria as a risk factor for cardiovascular disease. We too have found albuminuria to be associated with a $70-80 \%$ increased prevalence of cardiovascular disease [3] and a doubling of mortality risk (mostly cardiovascular in nature) [4] as compared to older people without microalbuminuria.

More recently, we and others have shown that albuminuria is associated with other diseases of older age. The two most outstanding diseases of older age associated with albuminuria are hip fractures and dementia. In one study of ours [5], a doubling of albuminuria was significantly associated with hip fracture risk in women (hazard ratio, 1.12, $95 \% \mathrm{CI}, 1.001-1.25$ ), but not in men. In two other studies, we found a borderline lower bone mineral density in association with albuminuria in men $[5,6]$. We also reported that participants with baseline albuminuria had a significantly increased risk of hip fracture compared with participants without albuminuria (adjusted hazard ratio $=1.36$ $[1.01,1.84$ ], $\mathrm{P}=0.05$ ) [7]. Last, it is known that up to $40-50 \%$ of people with hip fractures have cognitive impairment (such as dementia or mild cognitive impairment [a possible precursor of dementia). In our analyses we found that the association of cognitive impairment with hip fracture risk was attenuated with adjustment for the presence of albuminuria [8]. Stated differently, albuminuria played a role in mediating the association of hip fracture risk with cognitive impairment. Given the above findings, it would appear that albuminuria is more than a disorder of the glomerulus or a cardio-renal risk factor.

Several studies have shown albuminuria to be related to other disorders of aging. These include reduced physical performance, as measured by gait speed and grip strength [9]; dementia [10]; reduced lung function, as measured by one second forced expiratory volume [11]; microvascular cardiomyopathy [12]; abnormal reactivity of small [13] and large blood vessels [14]; and abnormal retinal findings in people with diabetes [15]. Taken together, these findings suggest that albuminuria may be an indicator of enhanced physiological aging beyond its known association with cardiovascular risk, and a possible marker of a systemic functional disorder of the microvasculature [16].

\section{Present Study}

We reasoned that if albuminuria is indeed a marker of aging or of enhanced susceptibility to illness, then hospitalization rates would be higher in people with albuminuria as compared to people without albuminuria. In addition, we hypothesized that rates of hospitalization would be increased as well for a wide variety of disorders, especially in certain domains of aging-for example, heart failure, injury, cancer and infection. Finally, given the adverse association of diabetes on health, we hypothesized that people with albuminuria and diabetes would have a higher rate of hospitalization than people with albuminuria but without diabetes. To do such an analysis, we examined the Cardiovascular 
Health Study (CHS) data set. CHS is a follow up study of nearly 6000 Caucasian and African American adults (age $\geq 65$ years at baseline) from four US research centers who have been passively followed for health outcomes over several decades. The cohort is well characterized and has served as a source for more than 1500 academic papers. Using incident hospitalization rates as a measure of outcome, we found albuminuria to have a pervasive and deleterious association on the health of older adults across a broad range of diagnoses. Hospitalization rates were 39\% higher among people with albuminuria compared to people without albuminuria, even after adjustment for hypertension and diabetes (the main determinants of albuminuria), and adjustment for concomitant estimated glomerular filtration rate. The number of hospital days was increased by $\sim 60 \%$ as well. Circulatory disorders (e.g., heart failure) accounted for the largest number of hospitalizations $(\sim 30 \%)$. Most hospitalizations, however, were not for circulatory disorders. Infectious, endocrine (e.g., diabetes), injury and respiratory (e.g., pneumonia) hospitalizations were increased 40$90 \%$. Cancer risks were also increased, but not significantly so. Genitourinary disorders, in particular, were increased by $\sim 40 \%$ in men and $\sim 75 \%$ in women. Diseases of the male genital organs (ICD 9 codes 600.0-608.0) were increased. Of interest, the presence of diabetes did not enhance the risk of being hospitalized for any of these disorders. From this latter finding it may be deduced that the effect of diabetes on hospitalization risk is mediated in large part through its association with albuminuria, not from diabetes per se. Also, of interest, was the fact that albuminuria did not significantly increase the risk of hospitalization for chronic kidney disease, though it did have a statistically significant association with acute kidney injury. For more specifics, the reader is referred to our article (see reference 1).

\section{Conclusion}

In conclusion, there are two take away messages from our study for the urologist and the nephrologist. One, albuminuria is most likely a manifestation of a systemic disorder that impacts the health of older adults. It is not just a renal microvascular disorder. Second, albuminuria is a risk factor for hospitalization. Additional attention to people with albuminuria, to optimize their health, may lead to cost savings.

\section{Acknowledgement}

None.

\section{Conflict of Interest}

No conflicts of interest.

\section{References}

1. Barzilay JI, Buzkova P, Shlipak MG, Bansal N, Garimella P, et al (2020) Hospitalization Rates in Older Adults with Albuminuria: The Cardiovascular Health Study. The Journals of Gerontology: Series A glaa020.
2. Garg AX, Kiberd BA, Clark WF, Haynes RB, Clase CM (2002) Albuminuria and renal insufficiency prevalence guides population screening: results from the NHANES III. Kidney Int 61(6): 2165-2175.

3. Cao JJ, Barzilay JI, Peterson D, Manolio TA, Psaty BM, (2006) The association of microalbuminuria with clinical cardiovascular disease and subclinical atherosclerosis in the elderly: the Cardiovascular Health Study. Atherosclerosis 187(2): 372-377.

4. Cao JJ, Biggs ML, Barzilay J, Konen J, Psaty BM, et al. (2008) Cardiovascular and mortality risk prediction and stratification using urinary albumin excretion in older adults ages 68-102: the Cardiovascular Health Study. Atherosclerosis 197(2): 806-813.

5. Barzilay JI, Bůžková P, Chen Z, de Boer IH, Carbone L, et al. (2013) Albuminuria is associated with hip fracture risk in older adults: the cardiovascular health study. Osteoporos Int 24(12): 2993-3000.

6. Fink HA, Vo TN, Langsetmo L, Barzilay JI, Cauley JA, et al. (2017) Association of Increased Urinary Albumin With Risk of Incident Clinical Fracture and Rate of Hip Bone Loss: the Osteoporotic Fractures in Men Study. J Bone Miner Res 32(5): 1090-1099.

7. Barzilay JI, Gao P, Clase CM, Mente A, Mann JF, et al. (2013) Albuminuria and rapid loss of GFR and risk of new hip and pelvic fractures. Clin J Am Soc Nephrol 8(2): 233-240.

8. Bůžková P, Barzilay JI, Fink HA, Robbins JA, Cauley JA, et al. (2014) Ratio of urine albumin to creatinine attenuates the association of dementia with hip fracture risk. J Clin Endocrinol Metab 99(11): 4116-4123.

9. Bůžková P, Barzilay JI, Fink HA, Robbins JA, Cauley JA, et al. (2019) Higher albumin:creatinine ratio and lower estimated glomerular filtration rate are potential risk factors for decline of physical performance in the elderly: the Cardiovascular Health Study. Clin Kidney J. 12(6): 788-794.

10. Barzilay JI, Fitzpatrick AL, Luchsinger J, Yasar S, Bernick C, et al. (2008) Albuminuria and dementia in the elderly: a community study. Am J Kidney Dis 52(2): 216-226.

11. Oelsner EC, Balte PP, Grams ME, Cassano PA, Jacobs DR, et al. (2019) Albuminuria, lung function decline, and risk of incident chronic obstructive pulmonary disease the NHLBI pooled cohorts study. Am J Respir Crit Care Med 199(3): 321-332.

12. Zobel EH, Winther SA, Hasbak P, on Scholten BJ, Holmvang L, et al. (2019) Myocardial flow reserve assessed by cardiac $82 \mathrm{Rb}$ positron emission tomography/computed tomography is associated with albumin excretion in patients with Type 1 diabetes. Eur Heart J Cardiovasc Imaging 20(7): 796-803.

13. Martens RJ, Henry RM, Houben AJ, Kallen Van der CJH, Kroon AA, et al. (2016) Capillary rarefaction associates with albuminuria: the Maastricht study. J Am Soc Nephrol 27(12): 3748-3757.

14. Seliger SL, Salimi S, Pierre V, Giffuni J, Katzel L, et al. (2016) Microvascular endothelial dysfunction is associated with albuminuria and CKD in older adults. BMC Nephrol 17(1): 82.

15. Barrett EJ, Liu Z, Khamaisi M, King GL, Klein R, et al. (2017) Diabetic Microvascular Disease: An Endocrine Society Scientific Statement. J Clin Endocrinol Metab 102(12): 4343-4410.

16. Stehouwer CDA (2018) Microvascular dysfunction and hyperglycemia: a vicious cycle with widespread consequences. Diabetes 67(9): 17291741 